\title{
MOVILIZACIÓN DEL SUELO EN TRES SISTEMAS DE PREPARO
}

Pedro Hurtado de Mendoza Borges ${ }^{1}$, Haroldo Carlos Fernándes ${ }^{2}$, Aloísio Bianchini ${ }^{3}$, João Carlos de Souza Maia ${ }^{4}$, Zaíra Morais dos Santos Hurtado de Mendoza ${ }^{5}$

\section{RESUMEN}

La acción de los implementos y máquinas utilizadas en la preparación del terreno puede causar perjuicios de gran magnitud debido a la falta o exceso de tratamiento de las camadas. En este trabajo se determinaron los índices de movilización para tres sistemas de preparo de un suelo ferralítico amarillo, sometido a la siembra directa durante los últimos cinco años con los cultivos de maíz y frijol. Los sistemas evaluados fueron el convencional y los conservacionistas cultivo mínimo y siembra directa. Se utilizaron un tractor de ruedas (modelo mf 265), un arado de discos, una grada niveladora, un escarificador, una sembradora-adobadora y un perfilometro de $2 \mathrm{~m}$. En el terreno se levantaron los perfiles natural, interno y final. Después se determinaron los índices área movilizada, área de elevación y ampollamiento, aplicándose el método de simpson para calcular las secciones transversales. Fue utilizado un delineamiento experimental en bloques casualizados con 4 repeticiones. Se observaron diferencias significativas entre los índices estudiados, presentando el sistema convencional los mayores valores. Los menores valores de área movilizada y de elevación correspondieron a la siembra directa, mientras el ampollamiento fue intermedio para este sistema. Se concluyó que el sistema de preparo influye sobre los índices de movilización y que hubo tendencia diferente de los mismos para cada sistema.

Palabras claves: perfil del terreno, ampollamiento, siembra directa.

\section{ABSTRACT}

\section{SOIL MOBILIZATION IN THREE TILLAGE SYSTEMS}

The action of implements and machines used for the soil prepare can cause losses of great magnitude due to lack or excess layers of treatment. In this study it was determined the rates of mobilization for three tillage systems of soil ferriferous-yellow, subjected to no-tillage during the last five years with crops of corn and beans. The evaluated systems were the conventional, the conservationists subsurface tillage and no-tillage. It was used a tractor wheels (model mf 265), a disk plow, a harrowing, a ripper, a seeder-fertilizer and a profilometer of $2 \mathrm{~m}$. It was raised the natural profiles, internal and finals on the ground. Then, it was determined the mobilized area index, elevation area and blistering, applying simpson's method to calculate the cross sections. It was used an experimental design in randomized blocks with 4 replications. It was observed significant differences between the indices studied, the conventional system featuring the highest values. The no-tillage system showed the lowest values for the areas of elevation and mobilized, however, the blistering was intermediate. It was concluded that the system of preparation influences the rates of mobilization and that there was a different trend for each system.

Keywords: soil profile, blistered, no-tillage system

Recebido para publicação em 05/10/2011. Aprovado em 03/10/2012.

1- Ingeniero Agrícola, Profesor Adjunto de la UFMT, Cuiabá - MT, pborges@ufmt.br

2- Ingeniero Agrícola, Profesor Asociado de la UFV, Viçosa-MG

3- Ingeniero Agrónomo, Profesor Adjunto de la UFMT, Cuiabá-MT

4- Ingeniero Agrónomo, Profesor Asociado de la UFMT, Cuiabá-MT

5- Ingeniera Forestal, Profesora Adjunta de la UFMT, Cuiabá-MT 


\section{INTRODUCCIÓN}

Las operaciones realizadas durante el preparo del suelo tienen como finalidad principal crear las condiciones necesarias para el desarrollo de las culturas. El preparo del suelo por el método convencional normalmente consiste en 2 etapas. En la primera etapa se trabaja hasta una profundidad de aproximadamente $30 \mathrm{~cm}$, utilizándose los arados o gradas pesadas y en la segunda, la movilización es mas superficial hasta aproximadamente $15 \mathrm{~cm}$, con una o más pasadas de grada niveladora. Este exceso de movilización asociado a las condiciones adversas de topografía y precipitaciones durante la época del preparo puede originar una acentuada deterioración físicoquímica y biológica de los suelos.

Con el propósito de reducir la movilización del suelo y paralelamente mantener cantidades razonables de restos vegetales sobre la superficie, surgieron nuevas tecnologías, como por ejemplo los sistemas conservacionistas. Esos sistemas tienen por finalidad disminuir el número de operaciones durante el preparo. Generalmente, se consideran el preparo reducido como cualquier método que mantenga un mínimo de $30 \%$ de residuos en la superficie después de la siembra, siendo los más utilizados, el cultivo mínimo con escarificador y la siembra directa (PEIXOTO et al., 1997).

Los escarificadores son implementos de astas usados durante el preparo primario de los suelos para romper la camada arable comprendida entre 15 y $30 \mathrm{~cm}$ (SILVEIRA, 1988). Sin embargo, Gadanha Júnior et al. (1991) definen el escarificador como un implemento que posibilita la desagregación del suelo en las camadas hasta la profundidad de $35 \mathrm{~cm}$. Esta práctica mecánica tiene por objetivo reducir la densidad del suelo y la resistencia a la penetración de raíces, aumentar la permeabilidad y disminuir el encharcamiento en terrenos planos, bien como romper las camadas superficiales incrustadas y camadas subsuperficiales compactadas (DALLA ROSA, 1981; KOCHHANN \& DENARDIN, 2000). Así, el escarificador ha caracterizado el sistema de cultivo mínimo.

Por otro lado, con la intensión de preservar el suelo y los recursos naturales surgió el sistema de siembra directa (DERPSCH et al., 1991; MELLO et al., 1998). La adopción de este sistema se intensificó con la fabricación de sembradorasadobadoras provistas de discos para el corte de restos vegetales remanecientes en la superficie y que también pueden penetrar en suelos compactados. La siembra directa consiste en la colocación de la semilla en suelo no preparado, lo que reduce la movilización y posibilita la permanencia de restos vegetales como cobertura del suelo. Para Branquinho et al. (2004), la siembra directa asociada a la rotación de culturas requiere menor cantidad de agua para el plantío y favorece el aumento de la actividad biológica del suelo debido al incremento de materia orgánica.

Evaluando los agregados de un Latosuelo Marrón distrófico durante 4 años en tres sistemas de preparo Sidiras et al. (1982) observaron mayor estabilidad en el sistema de siembra directa seguido por la escarificación y aradura. Esos resultados evidenciaron el efecto desagregador causado por la movilización del suelo durante su preparo. En ese trabajo se constató que después de la siembra directa, $67 \%$ de los agregados de la clase de 5-9 mm originalmente existentes, aún permanecieron estables en agua. Sin embargo, los agregados de esa clase para escarificación y aradura presentaron 23 y $3 \%$, respectivamente.

Carvalho et al. (2007) y (2008) cuantificaron la movilización del suelo causada por varios implementos agrícolas y observaron diferencias estadísticas en los resultados. Los implementos correspondientes al sistema convencional fueron responsables por la mayor movilización, seguidos por los empleados en el cultivo mínimo. Sin embargo, en esos estudios no fue considerada la movilización del suelo en el sistema de siembra directa.

Con base en la bibliografía consultada se verifica que los sistemas utilizados para preparar el suelo alteran su estructura físico-química y biológica, debido principalmente a la forma diferente de alcanzar y revolver las camadas más profundas. La hipótesis de que el grado de revolvimiento del suelo puede ser cuantificado para posterior comparación y análisis motivó la realización de esta investigación que tiene como objetivo determinar los indicadores de movilización en tres sistemas de preparo. 


\section{MATERIAL Y MÉTODOS}

El experimento se realizó durante dos años consecutivos, en un área perteneciente a la Universidad Federal de Viçosa, situada en la ciudad de Viçosa/MG, definida por las coordenadas geográficas $20^{\circ} 45^{\prime} 14^{\prime \prime}$ de latitud Sur y $42^{\circ} 52^{\prime} 53^{\prime \prime}$ de longitud Oeste y altitud media de $650 \mathrm{~m}$. En la región predomina el relevo montañoso (aproximadamente 85\%). El clima fue clasificado como Cwa, templado húmedo con veranos calientes e inviernos secos (KÖPPEN \& GEIGER, 1928). La temperatura anual media varia entre $14,0{ }^{\circ} \mathrm{C}$ e $26,1{ }^{\circ} \mathrm{C}$. El terreno fue sometido a la siembra directa durante los últimos cinco años con los cultivos de maíz y frijol de forma alternada.

En este trabajo se utilizaron los equipamientos e implementos siguientes:

- Tractor de ruedas, modelo MF 265, fabricado por la Massey Ferguson* con tracción delantera auxiliar, o sea, $4 \times 2$ (TDA), potencia nominal a $2200 \mathrm{rpm}$ de $47,8 \mathrm{~kW}(65 \mathrm{CV}) \mathrm{y}$ aproximadamente $3700 \mathrm{~kg}$ de lastre;

- Arado reversible de 3 discos de 28";

- Grada niveladora de doble acción "off-set" en la máxima abertura con 36 discos de 20 ";

- Escarificador de muelles constituido por 6 astas parabólicas con ancho de $30 \mathrm{~mm}$ y punteras de tipo común sin alas. Las astas fueron separadas a $45 \mathrm{~cm}$ y reguladas para una profundidad de $32 \mathrm{~cm}$, considerando el intervalo de 1,40 a 1,51 para la relación entre esas variables, sugerido por Borges et al. (1999);

- Sembradora-adobadora, modelo PC 2123, fabricada por la SEED MAX*. La máquina está formada por tres unidades de siembra separadas a $450 \mathrm{~mm}$, discos de corte lisos con diámetro de $406 \mathrm{~mm}$, mecanismos dosificadores de semilla de disco horizontal con orificios e diámetro de $356 \mathrm{~mm}$, mecanismos para aplicación de fertilizantes con discos de $356 \mathrm{~mm}$ de diámetro, surcadores de discos dobles e ruedas compactadoras lisas en forma de "V" para regulación de la profundidad con diámetro de $345 \mathrm{~mm}$;

- Perfilómetro con $2 \mathrm{~m}$ de largo, compuesto por una estructura metálica provista de base con nivel de burbuja, mural para fijar el papel o la cartulina y 80 agujeros para el deslizamiento de las varillas de $60 \mathrm{~cm}$ de largo dispuestas verticalmente y separadas en $25 \mathrm{~mm}$ una de la otra.

Conforme los implementos utilizados se definieron los tres sistemas de preparo siguientes:

- Convencional: Movilización inicial del suelo con el arado de discos y después dos pasadas de grada niveladora;

- Cultivo mínimo: Movilización del suelo con el escarificador y;

- Siembra directa: Movilización del suelo solamente en la línea de siembra con la propia sembradora-adobadora destinada al plantío directo.

Para evaluar el grado de revolvimiento del suelo se calcularon los indicadores de movilización propuestos por Gamero \& Lanças (1996), tales como: el área movilizada, el área de elevación y el ampollamiento.

* La citación de marcas no significa recomendación de los autores, sino la caracterización de los equipamientos utilizados.

El área movilizada es representada por la región plana comprendida entre el perfil natural (inicial) del suelo y el perfil de fondo originado por la acción del implemento, dada por:

$\mathrm{A}_{\mathrm{MOB}}=\mathrm{A}_{\mathrm{NAT}}-\mathrm{A}_{\mathrm{INT}}$

en que

$\mathrm{A}_{\mathrm{MOB}}=$ Área movilizada $\left(\mathrm{m}^{2}\right)$;

$\mathrm{A}_{\mathrm{NAT}}=$ Área correspondiente al perfil natural del suelo $\left(\mathrm{m}^{2}\right)$;

$\mathrm{A}_{\mathrm{INT}}=$ Área correspondiente al perfil de fondo del suelo $\left(\mathrm{m}^{2}\right)$.

El área de elevación es definida por la región plana situada entre el perfil de la superficie final del 
suelo y el perfil de fondo originado por la acción del implemento, dada por:

$\mathrm{A}_{\mathrm{ELE}}=\mathrm{A}_{\mathrm{FIN}}-\mathrm{A}_{\mathrm{NAT}}$

en que

$\mathrm{A}_{\mathrm{ELE}}=$ Área de elevación $\left(\mathrm{m}^{2}\right)$;

$\mathrm{A}_{\mathrm{FIN}}=$ Área correspondiente al perfil de la superficie final del suelo $\left(\mathrm{m}^{2}\right)$;

$\mathrm{A}_{\mathrm{NAT}}=$ Área correspondiente al perfil natural del suelo $\left(\mathrm{m}^{2}\right)$.

El ampollamiento del suelo es determinado por la relación siguiente:

$$
\mathrm{E}=\frac{\mathrm{A}_{\mathrm{ELE}}}{\mathrm{A}_{\mathrm{MOB}}} \cdot 100
$$

en que

$\mathrm{E}=$ Ampollamiento del suelo (\%);

$\mathrm{A}_{\mathrm{ELE}}=$ Área de elevación $\left(\mathrm{m}^{2}\right)$;

$\mathrm{A}_{\mathrm{MOB}}=$ Área movilizada $\left(\mathrm{m}^{2}\right)$.

Los perfiles mencionados anteriormente fueron levantados con auxilio del perfilómetro para cada sistema de preparo del suelo. El perfil natural del suelo en las parcelas fue obtenido antes de realizar la operación, mientras que el correspondiente a la superficie final del suelo después del paso de los implementos o máquinas. Para levantar el perfil de fundo fue necesario retirar todo el suelo movilizado durante la operación.

Para garantizar que los tres perfiles descritos anteriormente fueran levantados en los mismos lugares y con igual nivel de referencia, en cada punto de colección se colocaron dos estacas de madera separadas una de la otra a 2,5 $\mathrm{m}$ de distancia para apoyar el perfilómetro. Las estacas fueron niveladas previamente en dirección perpendicular al desplazamiento de los conjuntos utilizados para preparar el suelo.

Los datos de cada perfil fueron procesados con auxilio de la planilla electrónica Excel para calcular las áreas por integración numérica utilizándose el método de Simpson, dado por:

$$
A=\int_{x_{0}}^{x_{n}} f(x) d x=\frac{h}{3} \cdot\left[P_{0}+P_{n}+4 \cdot \sum_{i=1}^{n / 2} P_{(2 \cdot i-1)}+2 \cdot \sum_{i=1}^{n / 2-1} P_{(2 \cdot i)}\right]
$$

en que

$\mathrm{A}=$ Área de la región plana correspondiente al perfil $\left(\mathrm{m}^{2}\right)$;

$\mathrm{x}_{0}=$ Abscisa inicial del intervalo de integración (m); $\mathrm{x}_{\mathrm{n}}=$ Abscisa final del intervalo de integración (m); $\mathrm{f}(\mathrm{x})=$ Función continua que representa el perfil (m); $\mathrm{dx}=$ Diferencial da variable que representa el perfil (m); $\mathrm{h}=$ Distancia entre las varillas $(\mathrm{m})$;

$\mathrm{P}_{0}=$ Profundidad registrada en la lectura inicial del intervalo $(\mathrm{m})$;

$\mathrm{P}_{\mathrm{n}}=$ Profundidad registrada en la lectura final del intervalo $(\mathrm{m})$;

$\mathrm{P}_{(2 \mathrm{i}-1)}=$ Profundidad registrada en lecturas impares (m); $\mathrm{P}_{(2 \cdot \mathrm{i})}=$ Profundidad registrada en lecturas pares $(\mathrm{m})$; $\mathrm{n}=$ Número de varillas o cantidad de profundidades registradas (adimensional).

Para el planeamiento estadístico de la investigación se opto por el delineamento en bloques al acaso con cuatro (4) repeticiones, siendo cada sistema de preparo un tratamiento. Luego, fueron necesarias 12 unidades experimentales con $20 \mathrm{~m}$ de longitud y $5 \mathrm{~m}$ de ancho. Los indicadores de movilización de las 12 parcelas corresponden a la media de tres observaciones realizadas en las mismas.

La suposición de que los datos están normalmente distribuidos fue verificada con base en los gráficos de probabilidad, conforme sugerido por Montgomerry et al. (2004). Considerándose la subjetividad del método anterior, se aplicaron también las pruebas de Lilliefors, KolmogorovSmirnov y Shapiro Walk. La homogeneidad de varianza (homocedasticidade) se comprobó usándose las pruebas de Hartley, Cochran y Bartlett.

Cuando los datos no atendieron a las suposiciones de normalidad y homogeneidad, fueron transformados de acuerdo con las funciones propuestas por Banzato \& Kronka (1995); Ribeiro Júnior (2001). Solamente, cuando las referidas suposiciones fueron satisfechas, se procedió al análisis de varianza y a la comparación de medias de los tratamientos, conforme las pruebas de Fisher y Tukey, respectivamente. En los procedimientos

\section{REVENG}


gráficos y analíticos se utilizó el programa STATISTICA 8.0.

\section{RESULTADOS Y DISCUSIÓN}

En las Figuras 1, 2 y 3 se puede verificar que los sistemas de cultivo mínimo y siembra directa (sistemas conservacionistas) presentaron la mayor variabilidad en los perfiles de fundo y de la superficie final. Las irregularidades del perfil final asociada a la no inversión de la leva de suelo podría aumentar la resistencia a la erosión causada por las lluvias, sin embargo, la grande variación del perfil interno podría favorecer la compactación en camadas poco profundas, principalmente, en las regiones no alcanzadas por los órganos de trabajo. Conforme las mismas Figuras, el sistema convencional tiene la relación más uniforme entre las áreas movilizada y de elevación a lo largo de la sección transversal, pues la acción localizada de las astas y surcadores en los sistemas conservacionistas originó mayor fluctuación en la referida proporción.

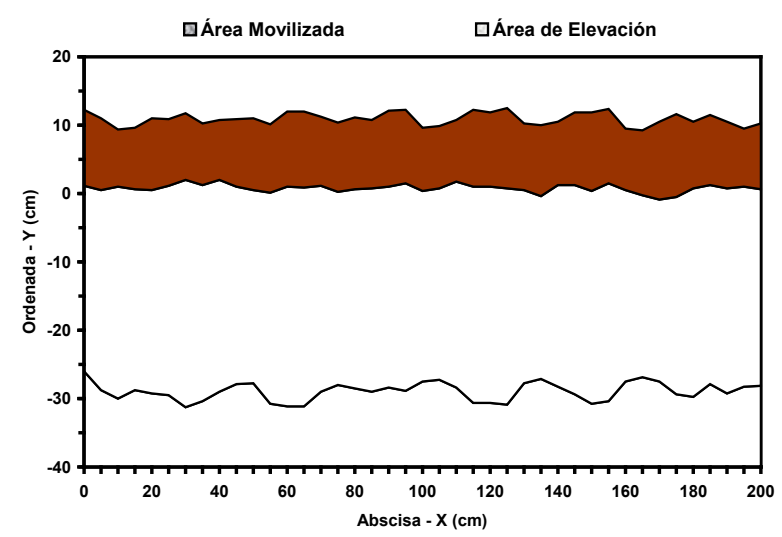

Figura 1. Representación de las áreas movilizada y de elevación en el sistema convencional.

Con base en las áreas representadas anteriormente, se determinaron los índices de movilización del suelo para cada método de preparo y seguidamente se verificaron las suposiciones de normalidad y homogeneidad de esos valores. Las referidas exigencias no fueron satisfechas, razón por la cual los datos se transformaron aplicándose varias funciones matemáticas. Para el área movilizada y de elevación la función logarítmica fue adecuada, mientras que para el ampollamiento se utilizó el arco seno de la raíz cuadrada, pues esos valores estaban expresos en porcentaje. Luego, se elaboraron los gráficos de probabilidad normal estandarizada, en función de los índices de movilización (Figuras 4, 5 y 6).

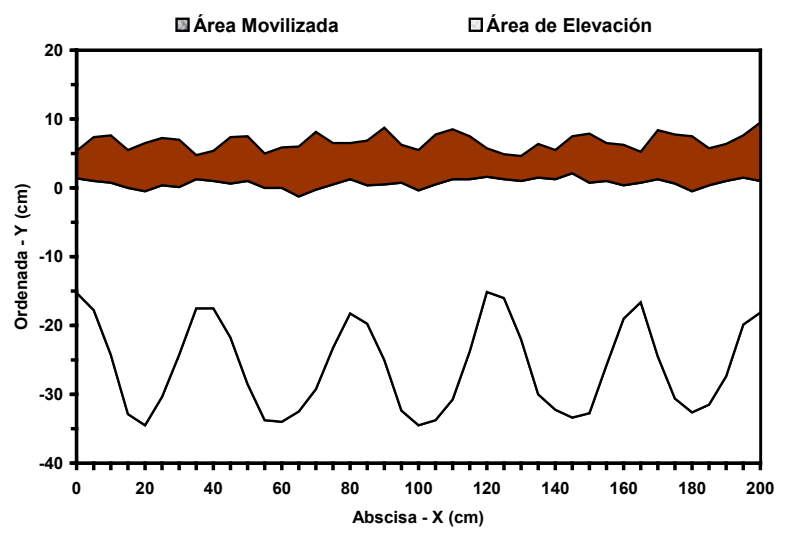

Figura 2. Representación de las áreas movilizada y de elevación en el sistema cultivo mínimo.

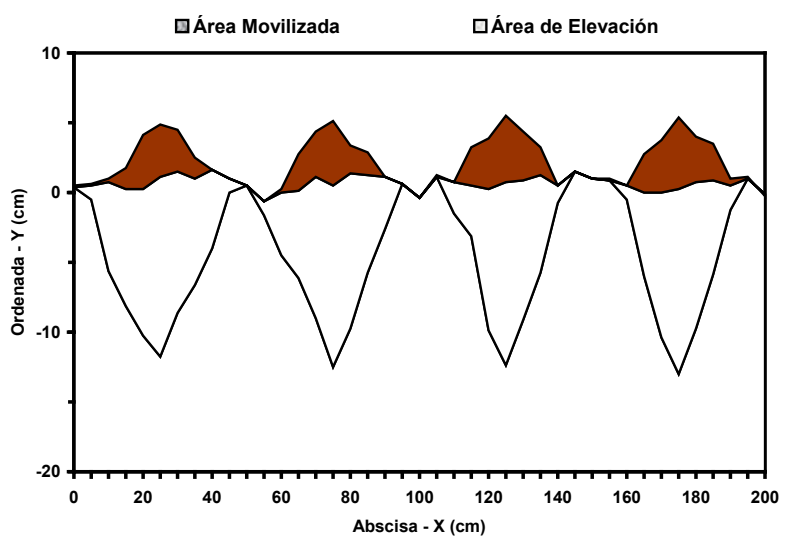

Figura 3. Representación de las áreas movilizada y de elevación en el sistema siembra directa.

Como puede ser observado en las Figuras 4, 5 y 6 , los valores transformados se encuentran próximos a la línea recta inclinada, indicando que los datos presentan distribución normal. Una vez que ese método es subjetivo, se consideró necesario verificar analíticamente, tanto la normalidad de los datos, cuanto la homogeneidad de varianzas de los tratamientos. Los resultados de esas pruebas se encuentran en los cuadros 1 y 2 . 


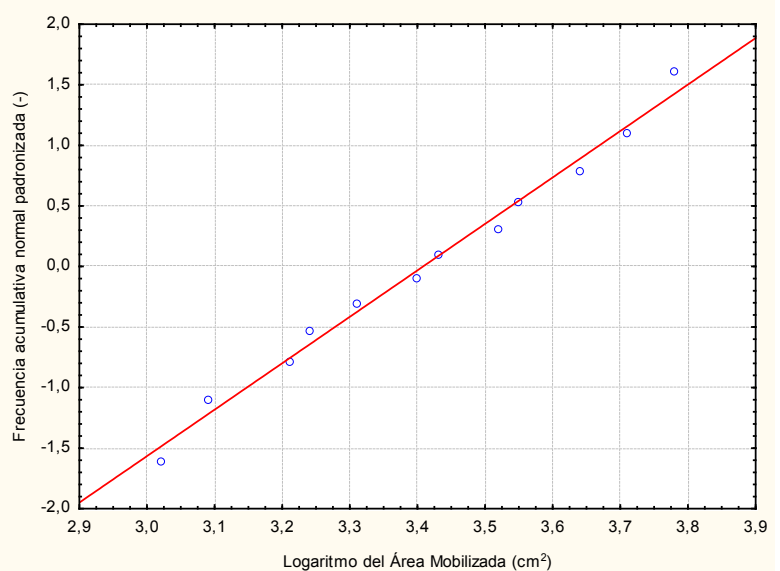

Figura 4. Probabilidad normal estandarizada para valores transformados del área movilizada.

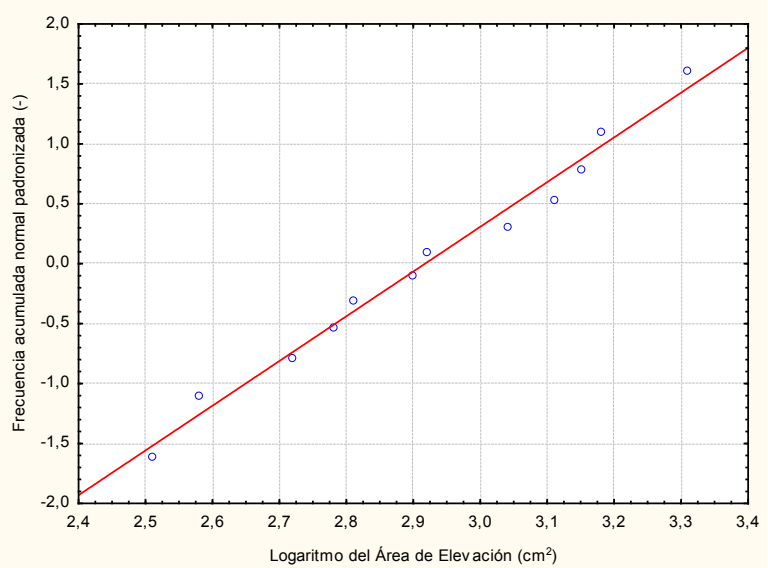

Figura 5. Probabilidad normal estandarizada para valores transformados del área de elevación.

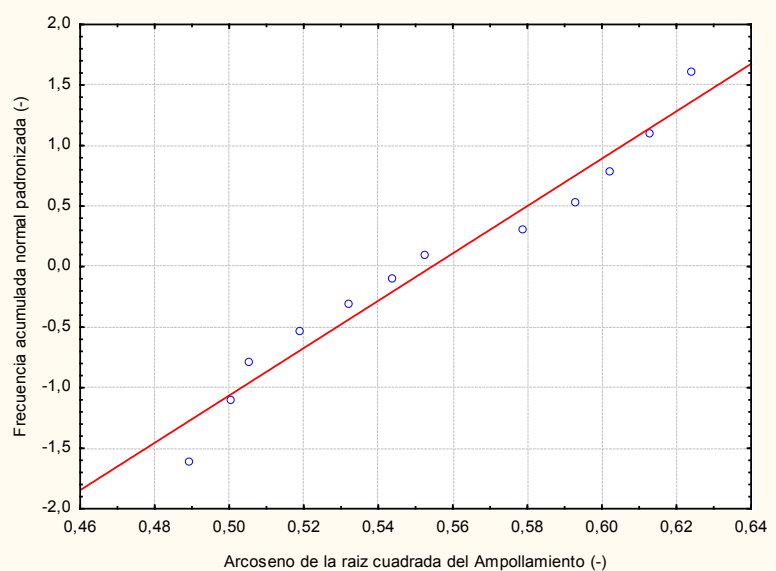

Figura 6. Probabilidad normal estandarizada para valores transformados del ampollamiento.

En el Cuadro 1 se constata que la probabilidad de aceitar la normalidad de los datos fue superior a $0,05(\mathrm{p}>5 \%$ ) para todos los índices de movilización, conforme todas las pruebas. También, se verificó que los resultados de las pruebas de Hartley, Cochran y Bartlett no fueron significativos. Luego, se concluyó que las hipótesis de normalidad de los datos y de homogeneidad de varianzas de los tratamientos no pueden ser rechazadas.

Después de atendidas las suposiciones de normalidad de los datos y homocedasticidade, se procedió al análisis de varianza, cuyo resumen se encuentra en el Cuadro 3. De acuerdo con ese Cuadro, los tratamientos o sistemas de preparo del suelo tuvieron efecto significativo, conforme la

Cuadro 1. Probabilidad de aceitar la hipótesis de normalidad de los datos

\begin{tabular}{cccc}
\hline \multirow{2}{*}{ Índice de movilización } & \multicolumn{3}{c}{ Prueba de normalidad } \\
\cline { 2 - 4 } & Kolmogorov-Smirnov & Lilliefors & Shapiro Walk \\
\hline Área movilizada & superior a 0,2 & superior a 0,2 & igual a 0,9441 \\
Área de elevación & superior a 0,2 & superior a 0,2 & igual a 0,9379 \\
Ampollamiento & superior a 0,2 & superior a 0,2 & igual a 0,4722 \\
\hline
\end{tabular}

Cuadro 2. Resumen con los resultados de las pruebas de homogeneidad de varianzas

\begin{tabular}{cccc}
\hline \multirow{2}{*}{ Índice de movilización } & Hartley & Cochran & Bartlett \\
\cline { 2 - 4 } & no significativo & no significativo & no significativo \\
Área movilizada & no significativo & no significativo & no significativo \\
Área de elevación & no significativo & no significativo & no significativo \\
Ampollamiento & & &
\end{tabular}

\section{REVENG}


prueba de Fisher al nivel de $1 \%$ de probabilidad. Los bajos coeficientes de variación muestran que hubo excelente precisión en la colección de los datos y adecuada homogeneidad entre las unidades experimentales.

Los valores medios de los indicadores utilizados para evaluar la camada de suelo alcanzada por los implementos en cada sistema de preparo, bien como los resultados de la prueba de Tukey $(\mathrm{p} \leq 0,01)$, se presentan en el Cuadro 4. En ese Cuadro se puede verificar que los menores valores en los índices de movilización correspondieron a los sistemas cultivo mínimo y siembra directa (conservacionistas). Esas diferencias evidencian el efecto significativo del sistema de preparo del suelo sobre los referidos índices.

Se puede comprobar que el área movilizada en el sistema convencional fue superior en relación a los conservacionistas, $11,48 \%$ y $81,97 \%$, respectivamente, en el cultivo mínimo y en la siembra directa. Para el área de elevación se constató, también, una tendencia semejante, siendo que los valores para el cultivo mínimo y la siembra directa fueron inferiores en $42,86 \%$ y $85,71 \%$. Sin embargo, el ampollamiento presentó comportamiento diferente, pues su valor en el sistema convencional fue mayor que los estimados para la siembra directa en $10,38 \%$ y en $34,68 \%$ para el cultivo mínimo (Cuadro 4). Los menores valores del ampollamiento para el cultivo mínimo y la siembra directa indican que esos sistemas atienden a las condiciones conservacionistas.

En la Figura 7 se representa la proporción de los índices de movilización, en función del sistema de preparo del suelo. Se puede constatar una relación directa entre el área movilizada y de elevación, es decir, a medida que el área movilizada aumenta, mayor es el incremento en el área de elevación. Sin embargo, el ampollamiento presentó tendencia diferente a la observada en esos dos indicadores. De cualquier forma, se confirma que el cultivo mínimo y la siembra directa revuelven menos el suelo. Se considera que la mayor movilización en el sistema convencional, asociado a la desprotección del suelo, favorece la degradación del terreno debido principalmente a la acción erosiva de lluvias y viento.

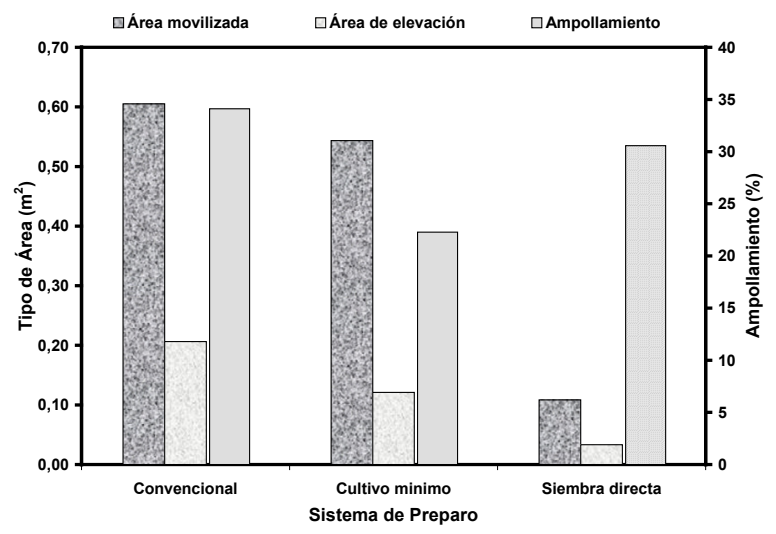

Figura 7. Índices de movilización del suelo para cada sistema de preparo.

Cuadro 3. Resumo del análisis de varianza con los valores de la estadística $\mathrm{F}$ y correspondiente significancia para los índices de movilización

\begin{tabular}{cccc}
\hline \multirow{2}{*}{ Fuente de variación } & \multicolumn{3}{c}{ Indicador de movilización } \\
& Área & Ampollamiento \\
\cline { 2 - 3 } & Movilizada & de elevación & \\
\hline Bloques & $3,96 \mathrm{~ns}$ & $0,09 \mathrm{~ns}$ & $4,76 \mathrm{~ns}$ \\
Tratamiento (Sistema de preparo) & $185695 * *$ & $47851 * *$ & $2798 * *$ \\
Coeficiente de variación $(\%)$ & 0,31 & 0,66 & 0,79 \\
\hline
\end{tabular}

"ns": no significativo (p>0,05); “*”: significativo $(p<0,05)$ e “**”: significativo $(p<0,01)$, conforme el teste Fisher.

Cuadro 4. Valores medios de los índices de movilización del suelo para los tres sistemas

\begin{tabular}{ccccc}
\hline \multirow{2}{*}{ Sistema de preparo } & \multirow{2}{*}{ Implemento } & \multicolumn{2}{c}{ Indicador de movilización y unidad } \\
\cline { 3 - 4 } & & \multicolumn{2}{c}{ Área $\left(\mathrm{m}^{2}\right)$} & Ampollamiento \\
\cline { 3 - 4 } & & Movilizada & de elevación & $(\%)$ \\
\hline Convencional & Arado e grade & $0,61 \mathrm{a}$ & $0,21 \mathrm{a}$ & $34,11 \mathrm{a}$ \\
Cultivo mínimo & Escarificador & $0,54 \mathrm{~b}$ & $0,12 \mathrm{~b}$ & $22,28 \mathrm{c}$ \\
Sembradura directa & Sembradora & $0,11 \mathrm{c}$ & $0,03 \mathrm{c}$ & $30,57 \mathrm{~b}$ \\
\hline
\end{tabular}

Obs. Para cada índice, en las columnas, medias seguidas de letras iguales no difieren entre si por el teste de Tukey ( $\mathrm{p} \leq 0,01$ ) 
Los resultados del área movilizada, en función del sistema de preparo no concuerdan con los obtenidos por Salvador et al. (1993). Los referidos autores estimaron menor valor de ese indicador para los arados de discos, en comparación al escarificador. Sin embargo, Carvalho et al. (2007) y (2008) no encontraron diferencias estadísticas significativas entre el área movilizada por el arado de discos y por el escarificador. Los valores presentados por ese autor fueron inferiores a los determinados en este estudio para el arado de discos y el escarificador, respectivamente, en $21,31 \%$ y 9,26\%. También, Cepik et al. (2010) obtuvo valores de área movilizada en el sistema de siembra directa inferiores en $33,77 \%$ a los de este trabajo. Esas discrepancias pueden ser debidas a las características geométricas de los implementos y órganos activos, al contenido de agua y materia orgánica en el suelo, bien como a la velocidad de operación. Luego, se considera que el referido indicador no es suficiente para evaluar el efecto del sistema en el preparo del suelo, pues la acción de los implementos y órganos puede ser diferente para valores semejantes de área movilizada y viceversa.

Salvador et al. (1993), Silva \& Gamero (1993), Carvalho et al. (2007) y (2008) no encontraron diferencias estadísticas significativas entre los valores del área de elevación para el arado de discos y el escarificador. El valor del área de elevación en esta investigación para el arado de discos fue $57 \%$ superior al estimado por Carvalho et al (2007), mientras, para el escarificador fue apenas $16,67 \%$ mayor al determinado por los referidos autores. Las divergencias observadas en relación a los trabajos presentados se deben, principalmente, al tamaño de los discos, tipo de astas y punteras del escarificador, distancia entre las astas y, probablemente, a la velocidad de avance del conjunto mecanizado.

Entre los valores de ampollamiento correspondientes al arado de discos y al escarificador, Salvador et al. (1993), Carvalho Filho (2007) no encontraron diferencias estadísticas significativas, lo que no concuerda con los resultados de la presente investigación. Los valores de ampollamiento estimados en este trabajo fueron superiores a los determinados por Carvalho Filho et al. (2007) en $47,23 \%$ y $12,03 \%$ para el arado y el escarificador, respectivamente. Como posibles causas para esas divergencias podemos citar la profundidad de trabajo, la forma y tipo de herramienta en los implementos, espacio entre los órganos activos, cantidad de materia orgánica y la velocidad de operación. Como el ampollamiento expresa la relación entre el área movilizada y la de elevación, puede ser un índice más completo para inferir sobre la movilización del suelo.

\section{CONCLUSIONES}

De acuerdo con los resultados obtenidos, se concluye que:

- Los sistemas de preparo tuvieron efecto sobre los indicadores de movilización del suelo;

- La tendencia de los valores del ampollamiento, en función del sistema de preparo del suelo, puede ser diferente a la observada en los otros dos indicadores de movilización;

- La irregularidad superficial presentada por el cultivo mínimo y la siembra directa indica que esos sistemas satisfacen las condiciones conservacionistas;

- El ampollamiento se evidenció como una importante medida para inferir sobre la degradación del suelo debido a la acción erosiva de las lluvias y del viento.

\section{REFERÊNCIAS BIBLIOGRÁFICAS}

BANZATTO, D.; KRONKA, S.N. Experimentação Agrícola. Jaboticabal: FUNEP, 1995. 247p.

BORGES, P.H.M.; BIANCHINI, A.; SABINO, M.H.C. Avaliação do trabalho realizado por escarificadores usando-se métodos analíticos. 
Engenharia Agrícola, Jaboticabal, v.18, n.3, p.93-1000, set.-dez. 1999.

BRANQUINHO, K.B.; FURLANI, C.E.A.; LOPES, A.; SILVA, R.P.; GROTTA, D.C.; BORSATTO, E.A. Desempenho de uma semeadora-adubadora direta, em função da velocidade de deslocamento e do tipo de manejo da biomassa da cultura de cobertura do solo. Engenharia Agrícola, Jaboticabal, v.24, n.2, p.374-380, mai.-ago. 2004.

CARVALHO FILHO, A.; CENTURION, J.F.; SILVA, R.P.; FURLANI, C.E.A.; CARVALHO, L.C.C. Métodos de preparo do solo: alterações na rugosidade do solo. Engenharia Agrícola, Jaboticabal, v.27, n.1, p.374-380, jan.-abr. 2007.

CARVALHO FILHO, A.; BONACIM, J.L.G.; CORTEZ, J.W.; CARVALHO, L.C.C. Mobilização de um latossolo vermelho acriférrico em função de sistemas de preparo do solo. Bioscience Journal, Uberlândia, v.24, n.3, p.1-7, jul.-sep. 2008.

CEPIK, C.T. C; TREIN, C.R.; LEVIEN, R.; CONTE, O. Força de tração e mobilização do solo por hastes sulcadoras de semadorasadubadoras. Revista Brasileira de Engenharia Agrícola e Ambiental, Campina Grande, v.14, n.5, p.561-566, 2010.

DALLA ROSA, A.D. Práticas mecânicas e culturas na recuperação de características físicas de solos degradados pelo cultivo- solo Santo Angelo (Latossolo Roxo Distrófico). 1981. 136f. Dissertação (Mestrado em Agronomia) - Universidade Federal do Rio Grande do Sul, Faculdade de Agronomia, Porto Alegre, 1981.

DERPSCH, R; ROTH, C.H.; SIDIRAS, N.; KOPKE, U. Controle da erosão no Paraná, Brasil: sistemas de cobertura do solo, plantio direto e preparo no conservacionista do solo. Eschborn: GTZ, 1991, 272p.
GADANHA JÚNIOR, C.D.; MOLIN, J.P.; COELHO, J.L.D.; YAHN, C.H.; TOMINORI, S.M.A. Máquinas e implementos agrícolas do Brasil. São Paulo: Instituto de Pesquisas Tecnológicas do Estado de São Paulo, 1991. $468 \mathrm{p}$.

GAMERO, C.A.; LANÇAS, K.P. Ensaio e certificação das máquinas de mobilização periódica do solo. In: MIALHE, L.G. Máquinas Agrícolas - Ensaios e Certificação. Piracicaba: FEALQ, 1996, p.463-514.

KOCHHANN, R.A.; DENARDIN, J.E. Implantación y manejo del sistema siembra directa. Passo Fundo: Embrapa -Trigo, 2000, $36 p$.

KÖPPEN, W.; GEIGER, R. Klima der Erde. Gotha: Verlag Justus Perthes. 1928.

MELLO, L.M.M.; FERREIRA, S.R.; YANO, E.H. Desempenho do equipamento sobre o desempenho do fragmento do Guandú (Cajanus cajan (L.) Millsp.). Ingeniería Rural y Mecanización Agraria en el Ámbito Latinoamericano. La Plata, Argentina, p.149153, 1998.

MONTGOMERY, D.C.; RUNGER, G.C.; HUBELE, N.F. Estatística aplicada à engenharia. Rio de Janeiro: LTC, 2004. 335p.

PEIXOTO, R.T.G.; AHRENS, D.C.; SAMAHA, M.J. Plantio direto: O caminho para uma agricultura sustentável. Ponta Grossa: IAPAR, 1997, 275p.

RIBEIRO, JÚNIOR, J.I. Análises Estatísticas no SAEG. Viçosa: UFV, 2001. 301p.

SALVADOR, N.; BENEZ, S.H.; BICUDO, S.J. Preparo periódico do solo I: desempenho operacional e mobilização do solo. In: CONGRESSO BRASILEIRO DE ENGENHARIA AGRÍCOLA, 22, 1993, Ilhéus. Anais... Ilhéus: CEPLAC/SBEA, 1993, v.3, p.1710-1720. 
SIDIRAS, N.; HENKLAIN, J.C.; DERPSCH, R. Comparação de três métodos de preparo do solo em relação a algumas propriedades físicas, perdas de solo e água e produtividade de soja e trigo em Latossolo roxo distrófico. Journal of Agronomy and Crop Science. Berlin, v.151, p.137-148, 1982.

SILVA, L.G., GAMERO, C.A. Efeitos de ordens de gradagens e de sistemas de aração sobre o desenvolvimento e produção do feijoeiro. In: CONGRESSO BRASILEIRO DE ENGENHARIA AGRÍCOLA, 22, 1993, Ilhéus. Anais... Ilhéus: CEPLAC/SBEA, 1993, v.3, p.1520-1535.

SILVEIRA, G.M. O preparo do solo: implementos corretos. Rio de Janeiro: Globo, 1988. 243p. 